Trauma Berufskrankh 2006 • 8[Suppl 3]:S296-S298 DOI 10.1007/s10039-005-1073-z

Online publiziert: 5. Januar 2006

(c) Springer Medizin Verlag 2006

E. Haider · Unfallkrankenhaus Berlin (ukb), Berlin

\title{
BG-Klinik 2010
}

\section{Zentrum des BG-Netzwerks zur Versorgung von Unfallverletzten}

Nach dem vom ukb formulierten Motto $-24 \mathrm{~h}$ - was auch geschieht - sind die BG-Kliniken in der Breite ihrer aufeinander abgestimmten Fachdisziplinen so aufgestellt, dass sie rund um die Uhr Unfallverletzte aller Schwierigkeitsgrade optimal in interdisziplinärer Weise behandeln können. Das große räumliche Einzugsgebiet der Kliniken belegt, dass sich die Kenntnis dieses Sachverhalts überregional verbreitet hat.

3. Kernkompetenz als Spezialeinrichtung für Komplikationsfälle in der Behandlung von Unfallverletzungen. Wenn andere Behandler und Behandlungseinrichtungen schwierige Fälle zur Behandlung in die BG-Klinik überweisen, ist dies als Anerkennung der hohen Qualität der in entsprechenden Fällen geleisteten Arbeit der BGKliniken zu betrachten.

4. Kompetenzbereich der Spezialisierung auf die stationäre und die ambulante Rehabilitation.

Akutversorgung und Rehabilitationsbehandlung sind in den BG-Kliniken vom Beginn der Behandlung an in idealer Weise miteinander verwoben. Auf diese Weise werden die Voraussetzungen für einen bestmöglichen und zügigen Rehabilitationserfolg geschaffen und ein Beitrag zur Kostendämpfung in der Sozialversicherung geleistet.

5. Kernkompetenz als Gutachteninstitut für komplexe Fragestellungen und als qualifizierte Beratungsstelle in allen Problemfällen mit verzögerten Heilund Rehabilitationsverläufen.

\section{Künftige Entwicklungen}

\section{Herausforderungen}

Für die Zukunft ist ein Erhalt des Status quo nicht ausreichend, sondern eine Wei- terentwicklung erforderlich. Die besonderen Kompetenzen einer BG-Klinik sind dabei ein solides Fundament, auf dem sich die Zukunft gestalten lässt. Allerdings müssen hierbei die Herausforderungen berücksichtigt werden, die sich durch die begonnenen Veränderungen in unserem gesamten Gesundheitswesen ergeben:

- DRG-Vergütungssystem

- Zwang zur Ausgabenbegrenzung im gesamten Gesundheitssystem

- Erwirtschaftung von Investitionen

DRG-Vergütungssystem. Die begonnene Entwicklung in Richtung einer nach landesweit einheitlichen Fallpauschalen erfolgenden Vergütung von Krankenhausleistungen hat zu einer verstärkten Ökonomisierung des Krankenhausgeschehens und in dessen Folge zu einer starken Reduzierung der Verweildauern der Patienten im Krankenhaus geführt. Diese Entwicklung geht mit einer Zunahme der ambulant erbrachten Krankenhausleistungen einher.

Erwirtschaftung von Investitionen. Die BG-Kliniken müssen künftig in viel stärkerem Maß als bisher Ersatz- und Neuinvestitionen, die zur Sicherung und Weiterentwicklung des Qualitätsniveaus erforderlich sind, aus dem laufenden Geschäftsbetrieb selbst erwirtschaften. In Zeiten knapper Kassen werden auch die Berufsgenossenschaften künftig die Mittel für Investitionen begrenzen. Zurzeit befindet sich ein Konzept zur Bildung eines Kapitalstocks zur zukünftigen Finanzierung der BG-Kliniken in der internen Abstimmung und wird hoffentlich bald umgesetzt.

\section{Lösungsmöglichkeiten}

Vernetzung mit geeigneten Leistungserbringern. Eine der wichtigsten Strategi- 
en, mit der eine BG-Klinik auf die genannten Herausforderungen reagieren sollte, ist die stabile Vernetzung mit geeigneten Leistungserbringern aller Versorgungsstufen, um

- auf diese Weise eine stetige Zuweisung von Patienten zur Behandlung in die BG-Klinik zu erreichen,

- kompetente Partner zu haben, in deren Hände die Patienten zum frühestmöglichen Zeitpunkt zur qualifizierten Weiterbehandlung überwiesen werden können und

- in der Lage zu sein, Ressourcen sparend standardisierte Verfahren zur Überwindung der Schnittstellenproblematik bei der Übergabe des Patienten in die Hände des Weiterbehandlers zu vereinbaren.

Letzteres ist ein wichtiges Anliegen der Berufsgenossenschaften.

\section{Ausweitung der ambulanten Angebote.} Die Ausweitung und Aktualisierung des ambulanten Leistungsangebots müssen nicht nur als Antwort auf die geänderte Vergütung stationärer Krankenhausleistungen erfolgen, sondern auch, um der zunehmenden Nachfrage der Patienten nach ambulanten Leistungen gerecht zu werden.

Einkaufskooperationen mit anderen Kliniken oder sonstigen Unternehmen. Sie sind eine weitere wichtige Teilantwort auf die Herausforderungen auf dem Gebiet des Beschaffungswesens und dienen dazu, günstigere Einkaufsbedingungen zu erhalten als ein einzelnes Krankenhaus. Auf VBGK-Ebene sind hier erste Initiativen gestartet worden. Je nach Beschaffungsgegenstand bieten sich aber auch andere Konstellationen an.

\section{Bisherige Maßnahmen des ukb}

Im Folgenden werden einige Aktivitäten angesprochen, die der Sicherung und dem Ausbau der Kernkompetenzen sowie der Vorbereitung auf künftige und gegenwärtige Herausforderungen dienen:

- Studien- und Forschungsprojekte

- Aus- und Weiterbildung von Fachärzten und medizinischen Hilfsberufen

Trauma Berufskrankh 2006 · 8[Suppl 3]:S296-S298

DOI 10.1007/s10039-005-1073-z

c) Springer Medizin Verlag 2006

E. Haider

\section{BG-Klinik 2010. Zentrum des BG-Netzwerks zur Versorgung von Unfallverletzten}

\section{Zusammenfassung}

Der vorliegende Artikel beschreibt aus der Sicht des Geschäftsführers einer BG-Klinik die Richtung der erforderlichen künftigen Entwicklung dieser Kliniken: Die BG-Klinik 2010 muss das Zentrum des BG-Netzwerks zur Versorgung von Unfallverletzten sein. Aufgrund ihrer Kernkompetenzen (Versorgung von Schwerstunfallverletzten, Traumazentrum zur interdisziplinären Versorgung von Unfallverletzten jeder Verletzungsschwere, Spezialeinrichtung für Komplikationsfälle, stationäre und ambulante Rehabilitation, Gutachteninstitut bzw. Beratungsstelle in allen Problemfällen) verfügen die BG-Kliniken über ein solides Fundament, auf dem sich die Zukunft gestal-

\section{BG Clinic 2010:. Center of the BG network for the care of trauma injuries}

\section{Abstract}

This article describes the directions which, in the opinion of the author, are necessary for the future development of a BG clinic: the BG clinic must be the center of the BG network for the care of trauma victims. Due to their basic areas of specialization (the treatment of severely injured, as trauma centers for the interdisciplinary care of all trauma victims, special arrangements for complication cases, in- and outpatient rehabilitation, as well as being centers for expert opinion and counseling) BG clinics will still exist after 2010. The challenges which must ten lässt. Herausforderungen, denen sie sich stellen müssen, sind u. a. das DRG-Vergütungssystem, der Zwang zur Ausgabenbegrenzung im gesamten Gesundheitssystem und die Erwirtschaftung von Investitionen. Antwortmöglichkeiten auf diese Herausforderungen werden dargestellt und konkrete Beispiele für Maßnahmen anhand der derzeitigen Aktivitäten des ukb gegeben.

\section{Schlüsselwörter}

BG-Netzwerk · Kernkompetenzen . DRG-Vergütungssystem .

Ausgabenbegrenzung · Wirtschaftlichkeit still be faced include the DRG-compensation system, the necessity to reduce costs in the health system generally, and the management of capital expenditure. Possible answers to these challenges are presented along with concrete examples for action from the current activities of the ukb.

\section{Keywords}

BG network - Core competence . DRG reimbursement system. Expenditure limitations · Economy 
BG-Netzwerk

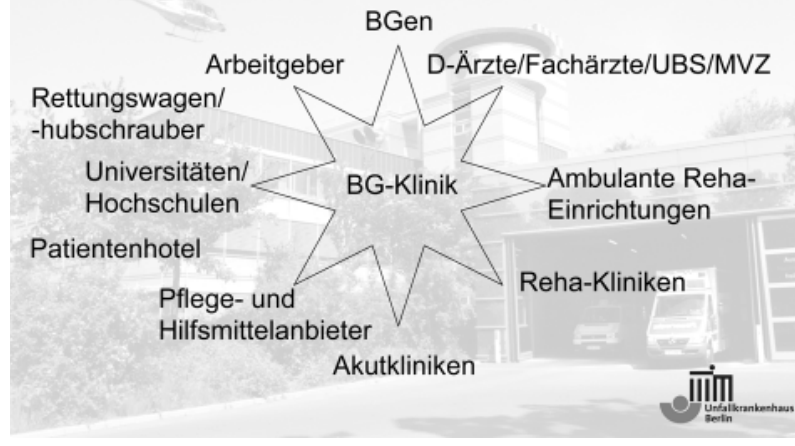

Abb. $1<$ Standort des ukb im BG-Netzwerk 2010

- Enge Kooperation mit den Hochschulen

- Enge Kooperationen mit anderen Krankenhäusern

- Enge Verzahnung der stationären mit der ambulanten Versorgung

- Konzentration auf das medizinische Kerngeschäft

Studien- und Forschungsprojekte. Das ukb hat sich seit Aufnahme seines Betriebs kontinuierlich mit deren Durchführung befasst. Auf diese Weise wird zum einen ein Beitrag zum medizinischen Fortschritt geleistet, zum anderem der Ruf des ukb durch die frühestmögliche Anwendung der daraus resultierenden "good practices" und das damit verbundene Setzen von Standards für andere Krankenhäuser als eine der führenden Einrichtungen auf dem Gebiet der Unfallmedizin gefestigt.

Aus- und Weiterbildung. Das ukb hat von Beginn an die Aus- und Weiterbildung von Fachärzten und medizinischen Hilfsberufen als ein wichtiges Instrument zur Gewinnung qualifizierten Nachwuchspersonals betrachtet. Angesichts des Nachwuchsmangels in den medizinischen Berufen wird dieses Instrument künftig eher noch wichtiger werden als bisher.

Kooperation mit Hochschulen. Das ukb pflegt eine enge Zusammenarbeit mit unterschiedlichen Universitäten und Hochschulen in Berlin und über Berlin hinaus. Sichtbarster Ausdruck ist die Tatsache, dass der ärztliche Direktor des ukb einen Lehrstuhl an der Ernst-Moritz-Arndt-Universität in Greifswald innehat.
Enge Kooperationen mit anderen Krankenhäusern. Damit verfolgt das ukb die Strategie, die Basis für eine qualitätsgesicherte und kosteneffiziente arbeitsteilige Zusammenarbeit in der medizinischen Leistungserbringung zu schaffen. Die Kooperationen erstrecken sich sowohl auf Akutkliniken unterschiedlicher Versorgungsstufen als auch auf Rehabilitationskliniken. Damit konzentriert sich das ukb auf seine spezifischen Stärken und strebt keine flächendeckende Ausweitung seines Leistungsspektrums an, sondern vernetzt sich mit qualifizierten Partnern, die auf ihrem Gebiet die Arbeit des ukb mit dem gleichen hohen Qualitätsanspruch ergänzen und fortsetzen oder umgekehrt selbst in Anspruch nehmen.

$\mathrm{Zu}$ diesem Zwecke haben Chefärzte Chefarztpositionen an anderen Kliniken übernommen und stellen so eine optimale Vernetzung miteinander in der stationären Versorgung sicher, z. B. durch die Übernahme der Befundung digitaler Röntgenbilder oder Computertomogramme im Wege der Teleradiologie.

\section{Verzahnung der stationären mit der am-} bulanten Versorgung. Im Hinblick auf die Phase zwischen der Entlassung aus der stationären Behandlung und der vollständigen Rückkehr in das häusliche Umfeld plant das ukb aktuell die Ansiedelung eines Patientenhotels in seiner Nähe. Operierte Patienten, deren pflegerische Versorgung nur noch in ganz geringem Maß notwendig ist, die jedoch noch der medizinischen Weiterbehandlung bedürfen, können frühzeitig in ein angenehmes Umfeld in der Nähe des ukb entlassen werden. von Kliniken des ukb in Personalunion
Dies ist im Hinblick auf die ökonomisch gebotene Verkürzung der stationären Verweildauer vorteilhaft und für die Patienten angenehmer als das weitere Verweilen auf der Krankenstation.

Das ukb hat sich seit seiner Eröffnung sehr intensiv um die Kooperation mit den niedergelassenen Durchgangsärzten und den Fachärzten im Einzugsgebiet gekümmert und setzt diesen Weg konsequent fort. In diesem Zusammenhang seien nur die regelmäßigen Fortbildungsangebote angeführt, die über die fachliche Zusammenarbeit und die Absprache von Verfahrensweisen mit dem Ziel der verzahnten Heilbehandlung und Rehabilitation hinaus die Kommunikation miteinander zum Wohle der Patienten fördern.

Konzentration auf das medizinische Kerngeschäft. Parallel zu den medizinisch-rehabilitativ ausgerichteten Aktivitäten in Richtung eines leistungsfähigen BG-Netzwerks zur Versorgung von Unfallverletzten um das ukb herum, verfolgen wir das Ziel der Konzentration der Arbeit auf das medizinisch-therapeutische Kerngeschäft und der Auslagerung von außerhalb dieses Kerngebiets liegenden Aufgaben auf andere Unternehmen. $\mathrm{Zu}$ letzteren sind Leistungen zur Hilfsmittelversorgung sowie die Sterilisation von im Krankenhaus verwendeten Instrumenten zu rechnen. Hinzu kommen weitere komplett in Auftrag gegebene Aufgaben, z. B. die Verpflegung der Patienten.

\section{Resümee}

Für das ukb als BG-Klinik 2010 ergibt sich der in - Abb. 1 gezeigte Standort im BGNetzwerk zur Versorgung von Unfallverletzten.

\section{Korrespondierender Autor Prof. Dr. E. Haider}

Unfallkrankenhaus Berlin, Warener Straße 7, 12683 Berlin E-Mail: haider@ukb.de

Interessenkonflikt: Es besteht kein Interessenkonflikt. Der korrespondierende Autor versichert, dass keine Verbindungen mit einer Firma, deren Produkt in dem Artikel genannt ist, oder einer Firma, die ein Konkurrenzprodukt vertreibt, bestehen. Die Präsentation des Themas ist unabhängig und die Darstellung der Inhalte produktneutral. 